\title{
Construction of a hepatocellular carcinoma cell line that stably expresses stathmin with a Ser25 phosphorylation site mutation
}

\author{
J. Du' , Z.H. Tao ${ }^{2}$, J. Li ${ }^{2}$, Y.K. Liư ${ }^{3}$ and L. Gan ${ }^{2}$ \\ ${ }^{1}$ Department of Chemistry, Baisc Medical College, Sichuan Medical University, \\ Luzhou, Sichuan, China \\ ${ }^{2}$ Research Center for Preclinical Medicine, Sichuan Medical University, Luzhou, \\ Sichuan, China \\ ${ }^{3}$ Liver Cancer Research Institute, Zhongshan Hospital, Fudan University, \\ Shanghai, China \\ Corresponding author: L. Gan \\ E-mail: gl-gump@163.com \\ Genet. Mol. Res. 14 (4): 12111-12117 (2015) \\ Received April 9, 2015 \\ Accepted July 10, 2015 \\ Published October 5, 2015 \\ DOI http://dx.doi.org/10.4238/2015.October.5.24
}

\begin{abstract}
We constructed hepatocellular carcinoma (HCC) cells that stably express stathmin with a Ser25 phosphorylation site mutation (stathmin S25A). We used the polymerase chain reaction for site-directed mutagenesis, constructed a stathmin S25A plasmid, and verified the results by restriction enzyme cleavage and sequencing technology. Using the liposome transfection method, stathmin wild-type and S25A HCCLM6 cells were established, which were identified by western blotting. The sequencing report of the stathmin S25A plasmid showed that stathmin serine at position 25 had mutated into alanine. Stable cells transfected with stathmin wild-type and S25A plasmids were constructed. Using western blotting, we confirmed that the expression level of stathmin pS25 in the stathmin S25A cells was reduced than that in the stathmin wild-type and HCCLM6 control cells $(P<0.05)$. We constructed stathmin S25A HCCLM6 cells, which offer an experimental model for further
\end{abstract}


investigation of the molecular mechanism of stathmin phosphorylation in hepatocarcinogenesis.

Key words: Site-directed mutagenesis; Hepatocellular carcinoma; Phosphorylation; Stathmin

\section{INTRODUCTION}

In vitro site-directed mutagenesis is one of the crucial techniques in the research of gene and protein structure and function. To further investigate the function of genes, manipulated point mutations in certain genes (including base substitutions, deletions, or insertions, etc.) are required for the observation of changes in gene function. Site-directed mutagenesis based on the polymerase chain reaction (PCR) is a time-saving and economic molecular biology approach that has developed rapidly over recent years. Site-directed mutagenesis can take place in any position of the DNA fragment, and it is now widely used in basic research regarding genes and protein structure or function (Sawano and Miyawaki, 2000; Young and Dong, 2003).

In our study, PCR site-directed mutagenesis was used in the construction of a recombinant plasmid with a site mutation at the Ser25 phosphorylation site of stathmin (stathmin S25A). The plasmid was then used to transfect the hepatocellular carcinoma (HCC) cell line HCCLM6 and establish cell lines that stably expressed wild-type stathmin or mutant S25A. The objective was to provide an experimental model for further studies on the molecular mechanisms of stathmin phosphorylation and its role in the pathogenesis of liver cancer.

\section{MATERIAL AND METHODS}

\section{Material}

The Flag-pcDNA3.1-stathmin wild-type (wt) plasmid was donated by Professor Baldassarre at Texas University; the HCC cell line HCCLM6 and the JM109 strain were preserved in our own laboratory. Restriction endonuclease HindIII (cat. ER0501) was obtained from Thermo Fisher Scientific Inc., Shanghai, China; KOD plus (KOD-201) and dNTP (cat. NTP-301) were bought from Bio Chemicals for Life Science, Shanghai, China; primers were synthesized by the Shanghai Biological Engineering Company. The gel extraction and plasmid extraction kits were bought from the TaKaRa Biotech Company; FLAG, stathmin, and anti-pS25 were bought from the Santa Cruz Company; other experimental reagents were imported and domestic analytical-grade products were used.

\section{Site-directed mutagenesis of eukaryotic expression vector of stathmin}

\section{Selection of site-directed mutagenesis site}

The 25th amino acid base site of Flag-pcDNA3.1-stathmin-wt was selected as the mutation site (changed from serine to alanine), and the primers were designed as follows. Stathmin-F: 5'-TGAGCTGATTCTCGCTCCTCGGTCAAAAG-3'; stathmin-R: 5'-CTTTTGACCGAGGAGCGAGA ATCAGCTCA-3'; stathmin-SF: 5'-CCCAAG CTTATGGATTACAAGGATGACGACGATAAGATGGC TTCTTCTGAT-3'; stathmin-SR: 5'-CGCGGATCCTCAGTCTCGTCAGCA-3'. 


\section{Determination of template DNA by PCR}

The concentration of Flag-pcDNA3.1-stathmin-wt was measured by PCR in a $50-\mu L$ reaction system. For the forward sequence of stathmin S25A, the following reaction mixture was used: $1 \mu \mathrm{L}$ KOD Plus, 10X $5 \mu \mathrm{L}$ PCR buffer, $5 \mu \mathrm{L}$ dNTP Mix, $1.5 \mu \mathrm{L}$ stathmin-SF, $1.5 \mu \mathrm{L}$ stathmin-R primers, $2.5 \mathrm{ng}$ template DNA, and $2 \mu \mathrm{L} \mathrm{MgSO}$, made up to $50 \mu \mathrm{L}$ with double-distilled $\mathrm{H}_{2} \mathrm{O}$. The amplification conditions for the PCR were: pre-denaturation at $95^{\circ} \mathrm{C}$ for $5 \mathrm{~min}$, then $95^{\circ} \mathrm{C}$ for $30 \mathrm{~s}$, $60.25^{\circ} \mathrm{C}$ for $30 \mathrm{~s}, 72^{\circ} \mathrm{C}$ for $15 \mathrm{~s}, 30$ cycles of amplification, and a final elongation at $72^{\circ} \mathrm{C}$ for $7 \mathrm{~min}$. For the reverse sequence of stathmin (S25A) the following reaction mixture was used: $1 \mu \mathrm{L}$ KOD Plus, 10X $5 \mu \mathrm{L}$ PCR buffer, $5 \mu \mathrm{L}$ dNTP Mix, $1.5 \mu \mathrm{L}$ stathmin-SF, $1.5 \mu \mathrm{L}$ stathmin-R primers, 2.5 ng template DNA, and $2 \mu \mathrm{LgSO}_{4}$, made up to $50 \mu \mathrm{L}$ with double-distilled $\mathrm{H}_{2} \mathrm{O}$. The amplification conditions for the PCR were: pre-denaturation at $95^{\circ} \mathrm{C}$ for $5 \mathrm{~min}$, then $95^{\circ} \mathrm{C}$ for $30 \mathrm{~s}, 65^{\circ} \mathrm{C}$ for $30 \mathrm{~s}$, $72^{\circ} \mathrm{C}$ for $24 \mathrm{~s}, 30$ cycles of amplification, and a final elongation at $72^{\circ} \mathrm{C}$ for $7 \mathrm{~min}$.

\section{Site-directed mutagenesis of stathmin}

The products from the PCRs described above were retrieved by gel extraction, and after the concentration was determined, a PCR was run in a 50- $\mu \mathrm{L}$ reaction system: $1 \mu \mathrm{L}$ KOD Plus, 10X $5 \mu \mathrm{L}$ PCR buffer, $5 \mu \mathrm{L}$ dNTP Mix, $1.5 \mu \mathrm{L}$ stathmin-SF, $1.5 \mu \mathrm{L}$ stathmin-R primers, $5 \mathrm{ng}$ of each anterior and posterior segments of stathmin S25A template, and $2 \mu \mathrm{L} \mathrm{MgSO}_{4}$, made up to $50 \mu \mathrm{L}$ with double-distilled $\mathrm{H}_{2} \mathrm{O}$. The amplification conditions were: pre-denaturation at $95^{\circ} \mathrm{C}$ for 5 min, then $95^{\circ} \mathrm{C}$ for $30 \mathrm{~s}, 60^{\circ} \mathrm{C}$ for $30 \mathrm{~s}, 72^{\circ} \mathrm{C}$ for $35 \mathrm{~s}, 30$ cycles of amplification, and a final elongation at $72^{\circ} \mathrm{C}$ for $7 \mathrm{~min}$.

1) The PCR products were digested with BamHI and HindIII; the reaction mixture comprised: $1 \mu \mathrm{g}$ PCR digestion products, BamHI and HindIII ( $1 \mu \mathrm{L}$ each ), 10X $1 \mu \mathrm{L} \mathrm{K}$ buffer, made up to $20 \mu \mathrm{L}$ with $\mathrm{H}_{2} \mathrm{O}$, which was reacted at $37^{\circ} \mathrm{C}$ for $1 \mathrm{~h}$. After gel extraction, $\mathrm{T}_{4} \mathrm{DNA}$ ligase was added for reaction at $16^{\circ} \mathrm{C}$ overnight.

2) Competent Escherichia coli JM109 was prepared and cultured at $37^{\circ} \mathrm{C}$ overnight. A monoclonal colony was picked and plasmid extraction was completed using the conventional alkaline lysis method. The extracted plasmid was identified by HindIII and BamHI restriction enzyme digestion and named FLAG-pcDNA3.1-stathmin S25A. The products described above were sent to Yingjun Biotechnology Company for sequencing, after which the recombinant plasmids with the correct sequence were extracted in large amounts.

\section{Screening and establishment of stably transfected cell clones}

Different concentrations of hygromycin were added to the culture systems of HCC cell line HCCLM6, which were of the same density. After incubating the cells at $37^{\circ} \mathrm{C}$ in $5 \% \mathrm{CO}_{2}$, we determined that the appropriate concentration of hygromycin was $400 \mu \mathrm{g} / \mathrm{mL}$.

Transfection of stathmin wt and S25A plasmid into HCCLM6 cells was performed according to the Lipofetamine ${ }^{\mathrm{TM}} 2000$ (cat. 11668-019, Thermo Fisher Scientific Inc., Shanghai, China). manufacturer instructions, and hygromycin was added $48 \mathrm{~h}$ after transfection for pressure screening.

Clones that went through the first round of screening were picked and digested for counting. After limiting dilution, the digested clones were transferred to a 96-well cell culture plate at a density of 1 cell per well. They were incubated at $37^{\circ} \mathrm{C}$ in $5 \% \mathrm{CO}_{2}$ until the clones were formed again. We then picked the well-grown clones for serial subcultivation and identification by western blotting. 


\section{RESULTS}

\section{Construction and identification of stathmin S25A point mutations in the recombinant plasmid}

The stathmin S25A recombinant plasmid was created by replacing the serine at the 25th amino acid with alanine by multiplex PCR. Screening transformation and sequencing were then performed and the Flag-pcDNA3.1 vector fragment (5480 bp) and the insert fragment (450 bp) were obtained through the digestion of the recombinant plasmid using restriction enzymes $\mathrm{BamHI}$ and HindIII (Figure 1A). The sequencing report showed that the insert sequence of the recombinant plasmid contained the expected mutation site (stathmin S25A) and the Flag label, while there was no mutation in the other amino acid sites. The mutation site is indicated by the circle in Figure 1B.

A

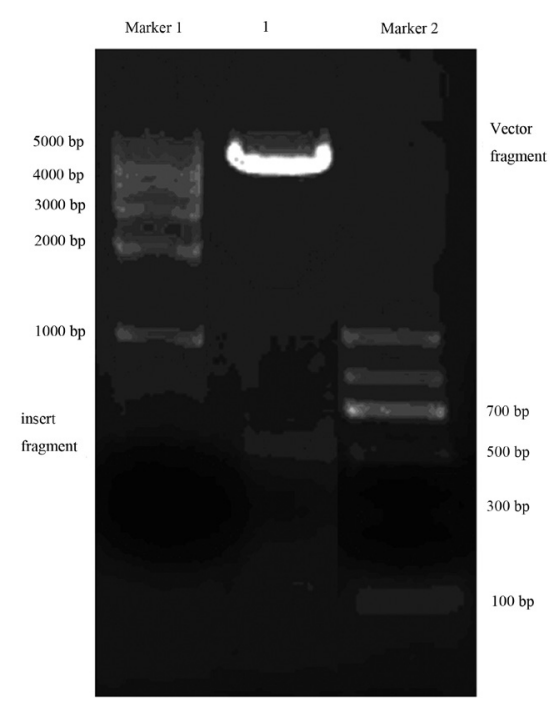

B

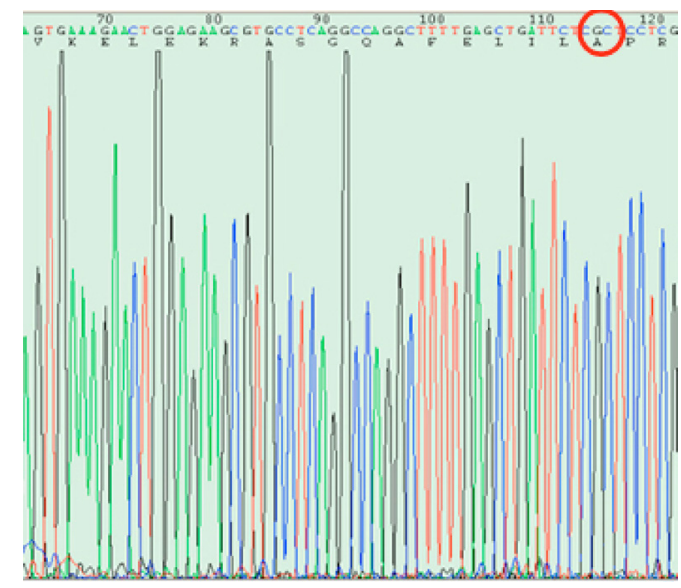

Figure 1. A. Plasmid restriction map of FLAG-pcDNA3.1-stathmin S25A; 1 was the S25A recombinant plasmid. B. Forward sequencing results of FLAG-pcDNA3.1-stathmin S25A (the 25th amino acid was mutated from serine to alanine). 


\section{Construction and identification of HCC cells line HCCLM6 with stable expression of wt and mutant stathmin plasmid}

After liposome transfection, limiting dilution, and monoclonal screening, HCCLM6 cells with stable expression of the Flag-pcDNA3.1 empty vector, stathmin wt, and stathmin S25A were derived, and marked as the control group, the stathmin wt group, and the stathmin S25A group, respectively. Total protein was extracted from the three groups for the detection of Flag expression by western blotting. The results showed that the relative expression of Flag was $0.48 \pm 0.09$, $0.52 \pm 0.09$, and $0.51 \pm 0.10$ in the control, stathmin wt, and stathmin S25A groups, respectively, implying that there was no significant difference in transfection efficiency among the three groups $(P>0.05)$. Further detection of stathmin expression and pS25 phosphorylation level showed that the relative expression of stathmin protein was $0.16 \pm 0.05,0.76 \pm 0.12$, and $0.43 \pm 0.01$, in the control, stathmin wt, and stathmin S25A groups, respectively, suggesting that the expression of stathmin was significantly upregulated in the stathmin wt and stathmin S25A groups $(P<0.01)$. The relative expression of stathmin pS25 protein was $0.19 \pm 0.04,0.41 \pm 0.04$, and $0.18 \pm 0.03$ in the control, stathmin wt, and stathmin S25A groups, respectively. This result shows that there was a significantly reduced expression of stathmin pS25 in the stathmin S25A group $(P<0.05)$, indicating the successful establishment of a stable cell line (Figure 2).
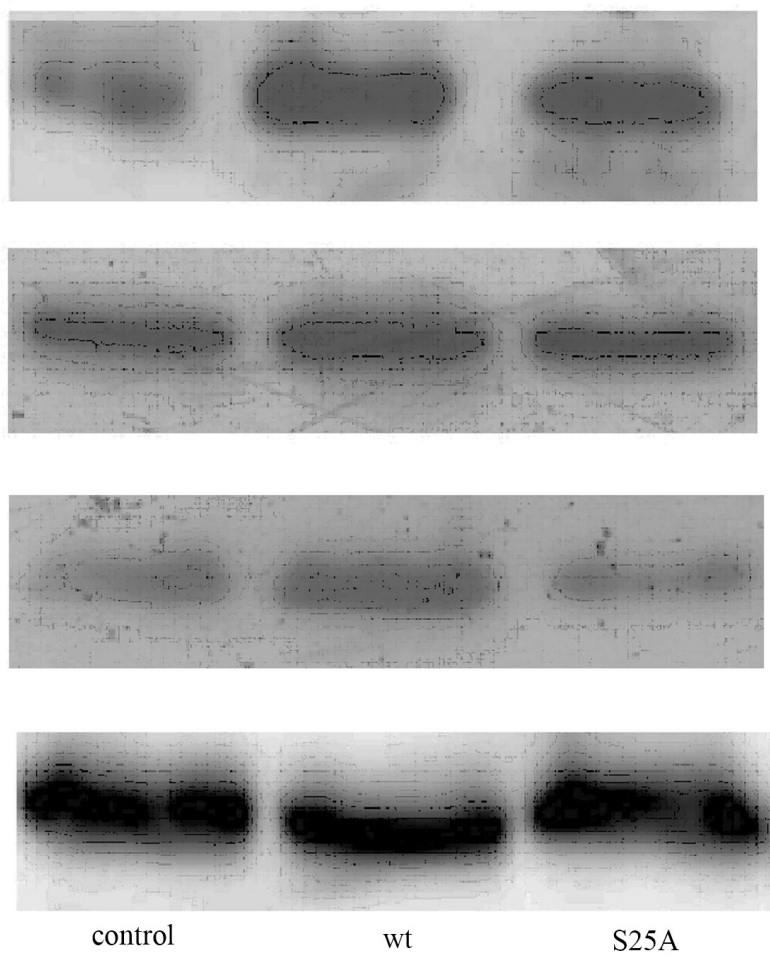

stathmin

Flag

stathminpS25

GAPDH

Figure 2. Expression of Flag, stathmin, and stathmin pS25 in the three groups (GAPDH is glyceraldehyde 3-phosphate dehydrogenase). 


\section{DISCUSSION}

Phosphorylation is an important post-translational modification that takes place widely in almost all biological activities. Numerous studies suggest that abnormalities in the phosphorylation process are closely related to tumor progression, indicating that changes in the phosphorylation state of proteins may be involved in tumorigenesis (Cicenas, 2008; Hutzen et al., 2009; Maclaine and Hupp, 2009; Nyalendo et al., 2009). Stathmin is a phosphorylation protein that is widely distributed in the cytoplasm and its function is to participate in microtubule dissociation and aggregation. There were four serine phosphorylation sites at the N-terminal of stathmin (Ser16, Ser25, Ser38, and Ser63), each regulated by a different protein kinase and playing different roles. Previous studies have detected the varying phosphorylation status of stathmin in HCC tissues at different sites, and found that when compared with non-metastatic liver cancer, there was a significant increase of stathmin expression in metastatic hepatic cancer, implying that the phosphorylation status of stathmin ser25 may be involved in the invasion and metastasis of HCC.

The construction of a HCC cell line with point mutations at the phosphorylation sites of stathmin wt and stathmin S25 is extremely important in the investigation of the correlation between stathmin ser25 phosphorylation and HCC development/progression. In the present study, we first used site-directed mutagenesis to construct a stathmin S25A recombinant plasmid (we changed the 25th amino acid of the Flag-pcDNA3.1-stathmin wt recombinant plasmid from serine to alanine), and sequencing was used to verify the correct mutation. In this experiment, we used the most economical and practical method, i.e., multiplex PCR, to produce the point mutations in stathmin. The key to this method is to design primers with the mutation point as the center, and to amplify the template by PCR. Two pieces of mutated sequence are then ligated after the removal of the template and the mutated sequences are amplified by PCR. Despite the multiple PCR and DNA purification recycling during the whole process, multiplex PCR is still a satisfying experimental approach owing to its low cost, the simple design of the primers, and its high success rate. In our study, we used Lipofetamine ${ }^{\mathrm{TM}} 2000$ liposome to transfect the successfully mutated recombinant plasmid into the HCC cell line HCCLM6. We then used limiting dilution and monoclonal screening to establish cell lines that stably expressed Flag-pcDNA3.1 empty vectors, stathmin wt, and stathmin S25A. Finally, by western blotting, we were able to detect the expression of Flag, stathmin, and stathmin pS25 in the three stable cell lines, thereby confirming the successful construction of the cell lines with stable expression of Flag-pcDNA3.1 empty vectors, stathmin wt, and stathmin S25A. Our study has provided a practical cell model for further investigations into: the impact of stathmin ser25 phosphorylation on the biological function of HCC cells (proliferation, apoptosis, invasion, etc.); the mechanisms of stathmin ser25 phosphorylation in the growth and metastasis of liver cancer in vitro and in vivo; and the molecular mechanisms of the phosphorylation of stathmin ser25 in the pathogenesis of liver cancer.

\section{Conflicts of interest}

The authors declare no conflict of interest.

\section{ACKNOWLEDGMENTS}

We received fundings from the Project of Sichuan Province Education Department (\#15ZA0161) and the Key Project of Sichuan Medical University. 


\section{REFERENCES}

Cicenas J (2008). The potential role of Akt phosphorylation in human cancers. Int. J. Biol. Markers 23: 1-9.

Hutzen B, Willis W, Jones S, Cen L, et al. (2009). Dietary agent, benzyl isothiocyanate inhibits signal transducer and activator of transcription 3 phosphorylation and collaborates with sulforaphane in the growth suppression of PANC-1 cancer cells. Cancer Cell Int. 9: 24.

Maclaine NJ and Hupp TR (2009). The regulation of p53 by phosphorylation: a model for how distinct signals integrate into the p53 pathway. Aging 1: 490-502.

Nyalendo C, Sartelet H, Barrette S, Ohta S, et al. (2009). Identification of membrane-type 1 matrix metalloproteinase tyrosine phosphorylation in association with neuroblastoma progression. BMC Cancer 9: 422.

Sawano A and Miyawaki A (2000). Directed evolution of green fluorescent protein by a new versatile PCR strategy for sitedirected and semi-random mutagenesis. Nucleic Acids Res. 28: e78.

Young $L$ and Dong $Q$ (2003). TAMS technology for simple and efficient in vitro site-directed mutagenesis and mutant screening. Nucleic Acids Res. 31: e11. 